\title{
Comparison of plan optimization for single and dual volumetric-modulated arc therapy versus intensity-modulated radiation therapy during post-mastectomy regional irradiation
}

\author{
LI-RONG ZHAO, YI-BING ZHOU and JIAN-GUO SUN \\ Cancer Institute of the People's Liberation Army, Xinqiao Hospital, Third Military Medical University, \\ Chongqing 400037, P.R. China
}

Received November 19, 2014; Accepted July 28, 2015

DOI: $10.3892 / \mathrm{ol} .2016 .4376$

\begin{abstract}
The aim of the present study was to investigate volumetric-modulated arc therapy (VMAT) with single arc (1ARC) and dual arc (2ARC), and intensity-modulated radiation therapy (IMRT), and to evaluate the quality and delivery efficiency of post-mastectomy regional irradiation. A total of 24 female patients who required post-mastectomy regional irradiation were enrolled into the current study, and 1ARC, 2ARC and IMRT plans were designed for each individual patient. The quality of these plans was evaluated by calculating the homogeneity index (HI), conformity index (CI) and specific volume dose to the ipsilateral lung, double lungs, contralateral breast, heart and spinal cord. For the delivery efficiency of these plans, the total treatment time (TTT) and the number of monitor units (MUs) were evaluated. The 1ARC and 2ARC VMAT plans exhibited significantly better HIs and CIs than IMRT. For dose-volume histogram analysis, 1ARC and 2ARC VMAT spared a more specific volume dose to the ipsilateral lung, double lungs, contralateral breast, heart and spinal cord than IMRT $(\mathrm{P}<0.05)$. A lower MU per 2.0-Gy fraction was required for $1 \mathrm{ARC}$ (539 MU) and 2ARC (608 MU) than for IMRT (1,051 MU). Thus, TTT was correspondingly reduced in $1 \mathrm{ARC}$ and $2 \mathrm{ARC}$ compared to IMRT $(\mathrm{P}<0.05)$. There was no significant dose-volume difference in all the organs at risk (OARs) between the 1ARC and 2ARC plans $(\mathrm{P}>0.05)$, and 2ARC VMAT displayed a better HI and $\mathrm{CI}$ than 1ARC VMAT $(\mathrm{P}<0.05)$. By contrast, 1ARC VMAT was superior to $2 \mathrm{ARC}$ VAMT with regard to MU and TTT $(\mathrm{P}<0.05)$. The 1ARC and 2ARC VMAT plans demonstrated significantly better dose distribution in a shorter treatment
\end{abstract}

Correspondence to: Mr. Jian-Guo Sun, Cancer Institute of the People's Liberation Army, Xinqiao Hospital, Third Military Medical University, 183 Xinqiao Street, Chongqing 400037, P.R. China E-mail: sunjg09@aliyun.com

Key words: volumetric-modulated arc therapy, intensity-modulated radiation therapy, mastectomy, regional irradiation, plan optimization time than IMRT for post-mastectomy regional irradiation, and spared the majority of OARs without compromising target coverage. The results of the present study suggest that 2ARC VMAT may be an alternative to $1 \mathrm{ARC}$ in order to obtain a more optimal HI and CI.

\section{Introduction}

It has been proven that regional radiotherapy, an integral part of breast-conserving therapy or mastectomy, can reduce the risk of local-regional recurrence of breast cancer and improve overall survival (1-5). Conventionally, post-mastectomy regional irradiation is planned using the two-dimensional data of the central axis of the treatment field and the wedged tangential pair treatment beams (6-9). More recently, intensity-modulated radiation therapy (IMRT), which has the ability to improve dose conformity and to decrease doses to the surrounding critical healthy structures (10-12), has been applied in post-mastectomy regional irradiation. However, 'step-and-shoot' IMRT is far from perfect in the clinical setting, and the development of novel radiotherapy techniques and devices is required. The optimization of a radiotherapy plan, the sparing of healthy tissues and the reduction in treatment time during the radiotherapy workflow are crucial.

Capable of delivering a highly conformal dose distribution in a relatively shorter duration, volumetric-modulated arc therapy (VMAT) (13) has aroused enormous interest in radiotherapy institutes worldwide. VMAT has been previously compared with IMRT for the treatment of various types of cancer at different sites (14-19). Although it has been well established that VMAT results in improved delivery efficiency over IMRT (20), there are few studies on whether VMAT represents a better treatment plan than IMRT for post-mastectomy regional irradiation.

In the present study, VMAT plans with single arc (1ARC) and dual arcs (2ARC) were evaluated and compared to step-and-shoot IMRT technology with regard to plan quality parameters, including homogeneity/conformity index (HI/CI), mean dose to the contralateral breast, ipsilateral lung and heart, and the maximum dose to the spinal cord, as well as the treatment efficiency parameters, including total treatment time (TTT) and number of monitor units (MUs). 


\section{Materials and methods}

Patient selection and image acquisition. A total of 24 female patients who required post-mastectomy regional irradiation in the Department of Oncology, Xinqiao Hospital (Third Military Medical University, Chongqing, China) between May and October 2013 were selected for enrolment. Patients aged between 18 and 75 years, with an Eastern Cooperative Oncology Group score of 0-2 (21) and adequate function of the liver, kidney, heart and hematopoietic system were considered eligible for the study. Patients that had undergone radiotherapy of the axillary or supraclavicular lymph nodes were excluded from the study. Among the 24 enrolled patients, there were 11 cases of right-sided breast cancer and 13 cases of left-sided breast cancer. The median age of the patients was 54 years (range, 21-74 years). All cases involved ductal or lobular carcinoma in different quadrants and underwent mastectomy. The stages ranged from pT3N2M0 to pT4N3M0, and required post-mastectomy regional radiotherapy according to National Comprehensive Cancer Network guidelines (2013, V1) (22). Following immobilization of the patients with a thermoplastic body-fix frame in a supine on the treatment bed, the patients were scanned using a helical computed tomography (CT) scanner with a 5-mm slice thickness. Scan extension included the underside of the chin to the upper abdomen. The CT data were transferred to the commercial treatment planning system (TPS), Oncentra MasterPlan V4.1 (OMP; Nucletron $\mathrm{BV}$, Veenendaal, The Netherlands), for contouring of targets and plan design. Written informed consent was obtained from all patients and the study was approved by the Medical Ethics Committee of Xinqiao Hospital, Third Military Medical University.

Definition of target volume and organs at risk (OARs). The radiotherapy physicians and planners used a standardized set of contouring guidelines (23). Target volumes and OARs were delineated and confirmed by three different qualified radiotherapy physicians. The clinical target volume (CTV) encompassed the post-operated chest wall, supraclavicular lymph nodes and internal mammary nodes. CTV was defined medially at the lateral edge of the sternum, including the sternal-rib junction, laterally at the preexisting breast tissue, including the mid axillary line, inferiorly at the inframammary fold and superiorly at the inferior edge of the cricoid cartilage. The medial extent of the mastectomy scar was typically included. Plan target volume (PTV) was obtained by expanding $3 \mathrm{~mm}$ in all directions except toward the skin. The border of the PTV on the skin side was at least $1 \mathrm{~mm}$ from the skin surface. The surrounding critical healthy tissues, including the contralateral breast, ipsilateral lung, spinal cord and heart, were contoured. An extra 3-mm margin was added to the spinal cord as the planning organ-at-risk volume (PRV).

Planning objective. All subsequent plans were conducted on OMP. For all patients, the prescribed dose was set to deliver 50 Gy normalized to $100 \%$ in 25 fractions encompassing at least $95 \%$ of the PTV, i.e., $\mathrm{V}_{100 \%}>95 \%$, while $\leq 2 \%$ of any PTV would receive $>110 \%$ of its prescribed dose; the PTV ranged from 269.00 to $783.00 \mathrm{~cm}^{3}$ (mean \pm standard deviation, $519.80 \pm 173.39 \mathrm{~cm}^{3}$ ). Other planning objectives for the OARs were as follows: Point dose of PRV of spinal cord, $<10 \mathrm{~Gy}$; percentage volume of ipsilateral lung receiving $>20$ Gy $\left(\mathrm{V}_{20}\right)$, $<20 \%$ and $30 \mathrm{~Gy}\left(\mathrm{~V}_{30}\right),<10 \%$; percentage volume of humerus receiving $>50 \mathrm{~Gy}\left(\mathrm{~V}_{50}\right),<5 \%$; and percentage volume of heart receiving $>40 \mathrm{~Gy}\left(\mathrm{~V}_{40}\right),<20 \%$. For the $\mathrm{T} 4$ patients with skin involvement, a bolus material with a thickness of $1 \mathrm{~cm}$ was added during the whole course, including immobilization, plan optimization, dosimetric calculation and radiation delivery.

Planning techniques. Using identical CT datasets and the exact same contours, all VMAT and IMRT plans were created using 6-MV photon beams commissioned for a Varian Trilogy Linac, equipped with a 120-leaf Multileaf Collimator (MLC; Varian Medical Systems, Palo Alto, CA, USA), with a maximal leaf speed of $2.5 \mathrm{~cm} / \mathrm{sec}$, a maximal jaw speed of $1.5 \mathrm{~cm} / \mathrm{sec}$, a maximal gantry speed of $6 \mathrm{~cm} / \mathrm{sec}$ and a variable dose rate of up to $600 \mathrm{MUs} / \mathrm{min}$. The optimization constraints and relative priorities were the same for the IMRT, and 1ARC and 2ARC VMAT plans. IMRT beams were equally spaced through the 200 sector angle in the axial plane, with fixed five gantry angles delivered in a step-and-shoot mode. 1ARC VMAT consisted of a single $200^{\circ}$ rotation, while $2 \mathrm{ARC}$ VMAT consisted of two coplanar arcs of $200^{\circ}$, optimized simultaneously and delivered with opposite rotation (clockwise and anticlockwise). The isocenter was placed at the center of the PTV. For the two VMAT plans, the couch angle was set as $0^{\circ}$ and at the same time, the collimator was set at $10^{\circ}$. The collapse cone photon dose calculation algorithm was used for all cases. The dose calculation grid was set at $2.5 \mathrm{~mm}$. Each plan was formulated by three different radiotherapy physicists and confirmed by three different radiotherapy physicians.

Evaluation parameters. The dose to the OARs, including the contralateral breast, ipsilateral lung, double lungs, spinal cord and heart, was measured and compared among the three planning techniques. The plans were evaluated according to dose-volume histogram (DVH) analysis, and plan quality was assessed by calculating the mean dose to the contralateral breast, ipsilateral lung, double lungs and heart, and the maximum dose to the spinal cord. The CI and HI were calculated according to the Radiation Therapy Oncology Group definition $(24,25)$ as follows: $\mathrm{CI}=\mathrm{V}_{\mathrm{RI}} / \mathrm{TV}$ and $\mathrm{HI}=\mathrm{I}_{\max } / \mathrm{RI}$, with $\mathrm{V}_{\mathrm{RI}}$ representing the volume $(\mathrm{V})$ of the prescribed dose for the PTV, TV as the total volume of the PTV, $\mathrm{I}_{\max }$ representing the maximum dose and RI as the prescribed dose of the PTV. These two formulae were therefore changed to $\mathrm{CI}=\mathrm{V}_{\text {Dprescribed }} / \mathrm{V}_{\mathrm{PTV}}$ and $\mathrm{HI}=\mathrm{D}_{\max } / \mathrm{D}_{\text {prescribed }}$. A CI and $\mathrm{HI}$ tendency towards a value of 1 demonstrated that the plan could be improved. Delivery parameters were recorded in terms of MU per fraction, mean dose rate, beam-on time, treatment time and TTT (defined as beam-on plus machine programming and setting time, but excluding patient positioning and imaging procedures).

Statistical analysis. All data presented in the text, tables and figures are presented as the mean \pm standard deviation. For 


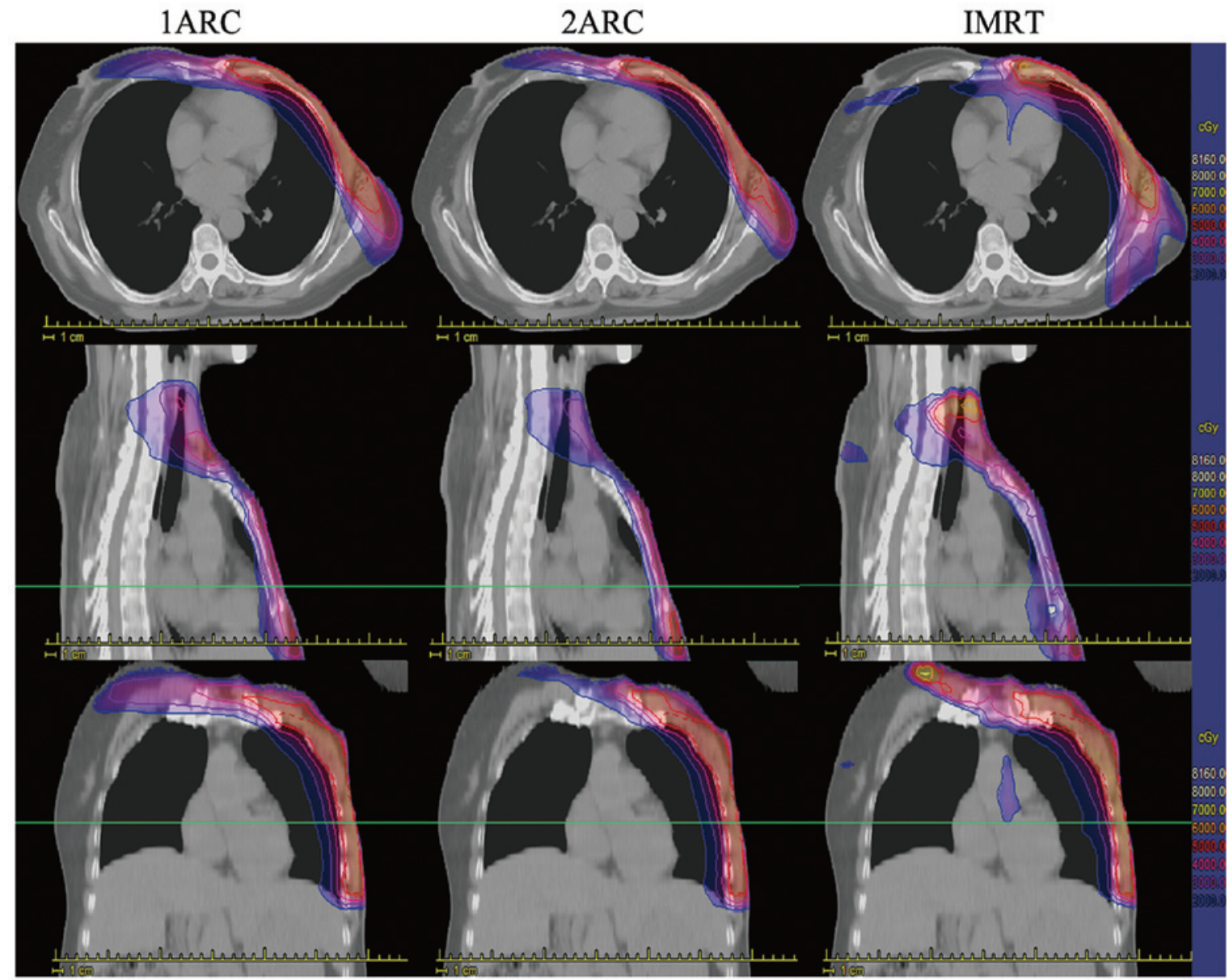

Figure 1. Isodose distributions on transverse, coronal and sagittal views of three treatment techniques in a representative case, planned by $1 \mathrm{ARC}$ and $2 \mathrm{ARC}$ volumetric-modulated arc therapy, and IMRT. 1ARC, single arc; 2ARC, dual arc; IMRT, intensity-modulated radiation therapy.

A

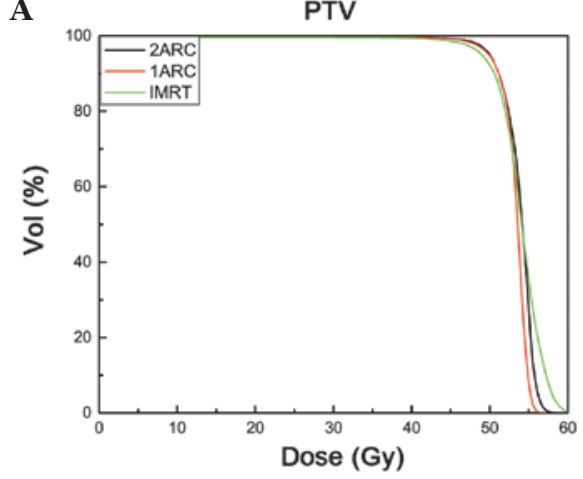

C

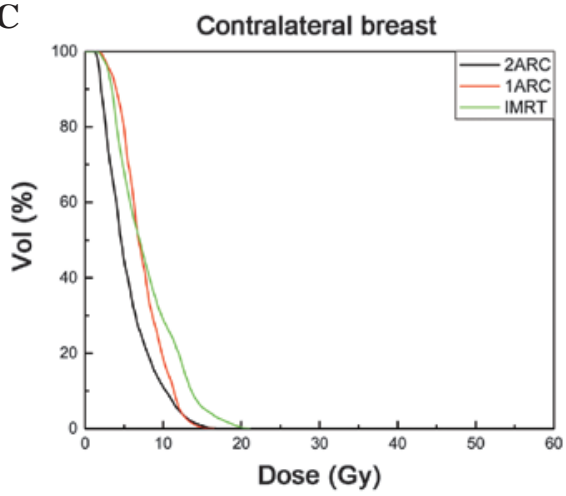

B

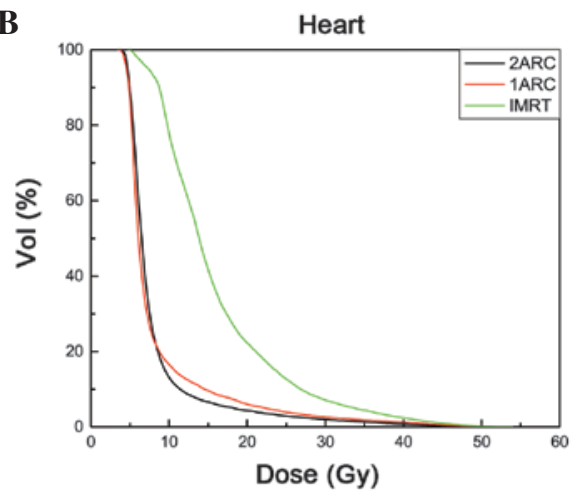

D

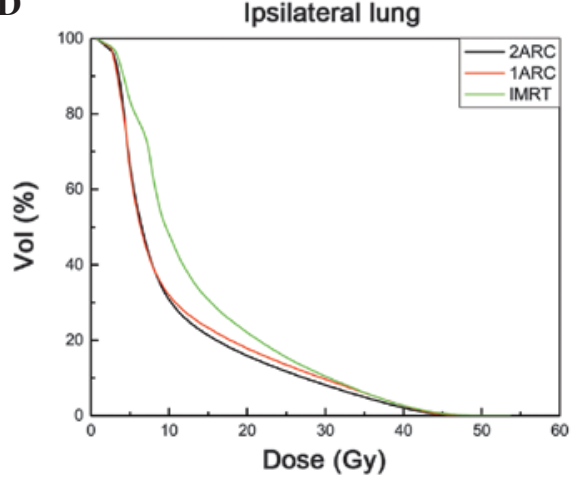

Figure 2. Mean cumulative dose volume histogram of the three treatment techniques for (A) planning target volume (PTV), (B) the heart, (C) the contralateral breast and (D) the ipsilateral lung. 1ARC, single arc; 2ARC, dual arc; IMRT, intensity-modulated radiation therapy. 
Table I. Target coverage and doses to organs at risk for three techniques (mean \pm standard deviation).

\begin{tabular}{|c|c|c|c|c|c|c|}
\hline Dosimetric parameters & IMRT & $1 \mathrm{ARC}$ & 2ARC & P-value ${ }^{a}$ & P-value ${ }^{b}$ & P-value \\
\hline Homogeneity index & $1.24 \pm 0.05$ & $1.20 \pm 0.06$ & $1.15 \pm 0.04$ & 0.005 & 0.005 & 0.005 \\
\hline Conformity index & $0.71 \pm 0.12$ & $0.78 \pm 0.05$ & $0.83 \pm 0.04$ & 0.005 & 0.005 & 0.005 \\
\hline $\mathrm{V}_{30 \text { (Ipsilateral lung) }}, \%$ & $10.3 \pm 3.3$ & $8.6 \pm 3.4$ & $8.2 \pm 3.9$ & 0.043 & 0.043 & 0.144 \\
\hline $\mathrm{V}_{20 \text { (Ipsilateral lung) }} \%$ & $22.0 \pm 4.6$ & $15.5 \pm 3.1$ & $17.7 \pm 4.1$ & 0.043 & 0.043 & 0.176 \\
\hline $\mathrm{V}_{5 \text { (Ipsilateral lung) }} \%$ & $83.0 \pm 10.9$ & $64.0 \pm 7.4$ & $65.2 \pm 6.7$ & 0.063 & 0.063 & 0.686 \\
\hline $\mathrm{V}_{30 \text { (double lungs) }}, \%$ & $5.9 \pm 1.6$ & $4.1 \pm 1.6$ & $3.8 \pm 1.6$ & 0.043 & 0.027 & 0.141 \\
\hline $\mathrm{V}_{20 \text { (double lungs) }}, \%$ & $14.5 \pm 5.8$ & $11.4 \pm 6.2$ & $10.3 \pm 5.7$ & 0.043 & 0.027 & 0.093 \\
\hline $\mathrm{V}_{5 \text { (double lungs) }}, \%$ & $52.9 \pm 8.2$ & $43.4 \pm 6.5$ & $43.5 \pm 6.7$ & 0.080 & 0.080 & 0.893 \\
\hline $\mathrm{V}_{10 \text { (contralateral breast) }}, \%$ & $28.9 \pm 3.2$ & $10.5 \pm 0.2$ & $10.8 \pm 0.1$ & 0.005 & 0.005 & 0.345 \\
\hline $\mathrm{D}_{15 \text { (contralateral breast) }}, \mathrm{Gy}$ & $13.7 \pm 0.2$ & $10.5 \pm 0.3$ & $8.9 \pm 0.3$ & 0.005 & 0.005 & 0.753 \\
\hline $\mathrm{D}_{\text {mean ipsilateral lung }}, \mathrm{Gy}$ & $9.5 \pm 2.1$ & $8.0 \pm 2.5$ & $7.9 \pm 2.2$ & 0.028 & 0.028 & 0.345 \\
\hline $\mathrm{D}_{\text {mean double lungs }}, \mathrm{Gy}$ & $7.3 \pm 11.6$ & $6.0 \pm 2.1$ & $6.5 \pm 16.6$ & 0.141 & 0.028 & 0.345 \\
\hline $\mathrm{D}_{\text {maxSpinalCord }}, \mathrm{Gy}$ & $9.6 \pm 4.3$ & $5.2 \pm 2.9$ & $4.8 \pm 2.7$ & 0.043 & 0.028 & 0.753 \\
\hline $\mathrm{D}_{\text {mean heart }}, \mathrm{Gy}$ & $10.6 \pm 5.0$ & $7.8 \pm 2.7$ & $7.2 \pm 2.3$ & 0.043 & 0.028 & 0.173 \\
\hline
\end{tabular}

${ }^{\mathrm{a}} 1 \mathrm{ARC}$ vs. IMRT; ${ }^{\mathrm{b}} 2 \mathrm{ARC}$ vs. IMRT; ${ }^{\mathrm{c}} 1 \mathrm{ARC}$ vs. $2 \mathrm{ARC}$. IMRT, intensity-modulated radiation therapy; $1 \mathrm{ARC}$, single arc; 2ARC, dual arcs; D, dose; V, volume.

Table II. Delivery parameters of three different treatment techniques (mean \pm standard deviation).

\begin{tabular}{lccccc}
\hline Delivery parameters & IMRT & 1ARC & 2ARC & P-value $^{\mathrm{a}}$ & P-value $^{\mathrm{b}}$ \\
\hline Monitor units & $1051 \pm 106$ & $539 \pm 88$ & $608 \pm 94$ & 0.005 & 0.005 \\
Beam-on time, sec & $285 \pm 19$ & $51 \pm 5$ & $125 \pm 7$ & 0.005 & 0.043 \\
Treatment time, sec & $440 \pm 56$ & $59 \pm 12$ & $129 \pm 34$ & 0.005 & 0.028 \\
Total treatment time, sec & $620 \pm 62$ & $180 \pm 16$ & $300 \pm 38$ & 0.005 & 0.005 \\
\hline
\end{tabular}

${ }^{\mathrm{a}} 1 \mathrm{ARC}$ vs. IMRT; ${ }^{\mathrm{b}} 2 \mathrm{ARC}$ vs. IMRT; ${ }^{\mathrm{c}} \mathrm{ARC}$ vs. $2 \mathrm{ARC}$. IMRT, intensity-modulated radiation therapy; $1 \mathrm{ARC}$, single arc; $2 \mathrm{ARC}, \mathrm{dual}$ arcs.

statistical analysis, exact Wilcoxon signed rank tests were used to assess any differences between the treatment parameters. Differences were considered statistically significant at $\mathrm{P}<0.05$. All computations were performed using the IBM SPSS statistics program (version 19; SPSS Inc., Chicago, IL, USA). All statistical tests were two-sided.

\section{Results}

Dose distribution. The typical axial, coronal and sagittal dose distributions produced by each of the three techniques in one representative case are displayed in Fig. 1. Fig. 2A demonstrates the mean DVH of the PTVs, comparing the three techniques for the entire cohort. Fig. 2B-D reports the average DVH computed for OARs, including the heart, contralateral breast and ipsilateral lung. The mean values of the study parameters among the three different techniques are tabulated in Table I, with their corresponding standard deviation.

Target coverage and dose homogeneity. All the plans were considered acceptable for at least the 95\% PTV volume that received $100 \%$ of the prescribed dose. The $1 \mathrm{ARC}$ and $2 \mathrm{ARC}$ VMAT plans resulted in a better dose HI compared with
IMRT, with a relative improvement of 7.8 and 3.3\%, respectively. This difference was significant $(\mathrm{P}<0.05)$. Similarly, the 1ARC and 2ARC VMAT plans exhibited a significantly higher CI than the IMRT (both $\mathrm{P}<0.05$ ) (Table I). As expected, the 2ARC VMAT plan produced the best dose CI compared with 1ARC VMAT and IMRT, with a relative improvement of $6.6 \%(P<0.01)$ and $15.7 \%(P<0.01)$, respectively. Similarly, the 2ARC VMAT plan demonstrated a significantly higher HI than the other two techniques (both $\mathrm{P}<0.01$ ) (Table II).

\section{OARs}

Spinal cord. All three techniques reached the planning objective of $\mathrm{D}_{\max }<10 \mathrm{~Gy}$ for the PRV of the spinal cord. The 1ARC and 2ARC VMAT plans led to a significant decrease in the $\mathrm{D}_{\max }$ of the spinal cord $(\mathrm{P}<0.05)$ compared with IMRT. No significant dose difference was found between the $2 \mathrm{ARC}$ and 1ARC VMAT plans.

Heart. For the 24 cases of post-mastectomy regional irradiation, the three techniques yielded low doses to the heart (Fig. 2B). The 1ARC and 2ARC VMAT plans resulted in a significantly lower heart dose than IMRT $(\mathrm{P}<0.05)$. No significant difference in the dose was found in the $2 \mathrm{ARC}$ and 1ARC VMAT plans. 
Contralateral breast. The 1ARC and 2ARC plans exhibited lower volume percentages for the contralateral breast that received 10 Gy compared with the IMRT plan $(\mathrm{P}<0.05)$. Furthermore, there was a lower dose for the contralateral breast that covered $15 \%$ of the volume compared with that detected by the IMRT plan $(\mathrm{P}<0.05)$ (Table I). No significant difference existed between $1 \mathrm{ARC}$ and $2 \mathrm{ARC}$ plans in terms of the volume percentage of the contralateral breast that received 10 Gy $(\mathrm{P}>0.05)$ or the dose of the contralateral breast that covered $15 \%$ of the volume $(\mathrm{P}>0.05)$.

Lung. The volume to the lung was evaluated, including ipsilateral and double lungs, and the specific dose limits were 5, 20 and $30 \mathrm{~Gy}$. It was evident that $1 \mathrm{ARC}$ and $2 \mathrm{ARC}$ VMAT resulted in a lower volume at an ipsilateral lung dose measuring 5, 20 and 30 Gy compared with IMRT (64.0 and 65.2 vs. $83.0 \%, 15.5$ and 17.7 vs. $22.0 \%$, and 8.6 and 8.2 vs. $10.3 \%$, respectively) (Table I). The volume differences were significant at 20 and $30 \mathrm{~Gy}(\mathrm{P}<0.05)$, but not at $5 \mathrm{~Gy}$ $(\mathrm{P}>0.05)$. Double lungs produced similar results to the ipsilateral lung. All the values are listed in Table I.

Planning and delivery efficiency. The mean values of MUs per fraction were 1,051 in IMRT, 539 in 1ARC VMAT and 608 in 2ARC VMAT (Table II). The 1ARC and 2ARC VMAT plans resulted in a 49 and $42 \%$ reduction in MUs per fraction consumed. The mean planning time was 6.9,23.4 and $32.5 \mathrm{~min}$ for IMRT, 1ARC and 2ARC, respectively. The mean time to prepare a VMAT plan was found to be approximately four times that required to prepare a fixed-field IMRT plan, which was attributed to the fundamental difference in the method for dosimetric optimization between the two techniques. The 1ARC and 2ARC plans exhibited a shorter treatment time, with a mean beam-on time of 51 and $125 \mathrm{sec}$, respectively, and the mean treatment time was 59, 129 and $440 \mathrm{sec}$ for 1ARC, 2ARC and IMRT, respectively. When considering the time for setup and position correction by image-guided radiotherapy, it was estimated that the TTT for each patient per fraction was 180,300 and $620 \mathrm{sec}$ for 1ARC, 2ARC and IMRT, respectively $(\mathrm{P}<0.05)$ (Table II). The 1ARC and $2 \mathrm{ARC}$ VMAT techniques reduced treatment time by $\sim 87$ and $40 \%$, respectively. As expected, 1ARC VMAT MU and TTT were less than those for 2ARC VMAT $(\mathrm{P}<0.05)$ due to the gantry rotation.

\section{Discussion}

Post-mastectomy regional irradiation improves disease management, while reducing patient toxicity. Improving the homogeneity of irradiation following mastectomy reduces the acute complication rate, as well as long-term fibrosis (26). The boosting of radiation to enhance the local control rate in high-risk women has previously been studied (27-29), and in consideration of this, reducing the excess irradiation to the contralateral breast is important, as it may decrease unnecessary cardiac and pulmonary irradiation.

The present study compared 1ARC, 2ARC and IMRT radiation plans in 24 cases of post-mastectomy regional irradiation. As three-dimensional conformal radiotherapy has not been demonstrated to be better than IMRT, the CI, HI and OAR dose were evaluated in IMRT, and $1 \mathrm{ARC}$ and $2 \mathrm{ARC}$
VMAT plans in the current study. Since treatment planning depended on variations in TPS, calculation algorithm, therapy equipment and most importantly, the skill level of the planner, the present study aimed to normalize the process of the three radiotherapy plans. For each patient, 1ARC, 2ARC and IMRT plans were generated under identical constraint conditions. Each plan was designed by three different radiotherapy physicists and confirmed by three different radiotherapy physicians. Thus, the interpersonal deviation was minimized

The results of the present study demonstrated that $1 \mathrm{ARC}$ or 2ARC VMAT significantly spared the OARs, without compromising target coverage when compared to the IMRT technique. Although the mean time to prepare a VMAT plan was roughly four times that of preparing a fixed-field IMRT plan, this plan displayed several inherent advantages, including a reduced treatment time and a decreased number of MUs. The greatest advantage of using VMAT in the current study was the shorter treatment time, which improved the comfort and satisfaction of the patients, while reducing the chance of movement due to discomfort and random errors introduced by intrafraction tumor motion.

In addition to the aforementioned application of VMAT, the present study compared the $1 \mathrm{ARC}$ and $2 \mathrm{ARC}$ technique in post-mastectomy regional irradiation. The advantages of using $2 \mathrm{ARC}$ were an increase in the modulation factor during optimization and the provision of more MLC control points than $1 \mathrm{ARC}$, achieving better dose distribution. However, in the current study, 2ARC did not exhibit significant advantages over 1ARC. The most notable and perhaps pertinent result was the further improvement in conformity and homogeneity indexes when using 2ARC VMAT. Treatment volume in the 2ARC plan could be much lower than that used in IMRT or 1ARC. Thus, 2ARC would direct less radiation to the OARs. The results of the present study are consistent with a previous study on nasopharyngeal carcinoma (14), in which 2ARC led to superior results in terms of dose coverage and spared OARs when compared with single arc VMAT, without sacrificing the delivery efficiency. Therefore, 2ARC VMAT may be an alternative to $1 \mathrm{ARC}$, allowing more optimal $\mathrm{HI}$ and $\mathrm{CI}$ values to be obtained, since 2ARC VMAT created a wide range of beam incidents that allowed a larger degree of freedom in the inverse optimizing process, and a greater capability for modulating the gantry speed, MLC shape and dose rate.

In conclusion, the advantage of delivering better dose distribution in a shorter treatment time (14-19), afforded by 1ARC and 2ARC VMAT, allowed these therapies to serve as more efficient methods of delivering post-mastectomy irradiation than the fixed-field IMRT technique. Long-term follow-up could provide actual toxicity profiles and a clear assessment of VMAT treatment efficacy. Furthermore, the present study did not include patients who underwent breast-conserving radiotherapy, and further studies could focus on these patients to provide a greater insight into post-mastectomy irradiation. The 1ARC and 2ARC VMAT plans produced superior dose distribution for post-mastectomy regional irradiation with less dose exposure to critical healthy structures. The plan quality and delivery efficiency made VMAT a reasonable option for post-mastectomy regional irradiation, while the 2ARC VMAT resulted in an improved $\mathrm{HI}$ and CI compared with the $1 \mathrm{ARC}$ plan. 


\section{Acknowledgements}

The authors are grateful for the support of the National Natural Science Foundation of China (grant no. 81272910) and the Clinical Foundation of the Third Military Medical University, Chongqing, China (grant no. 2011XLC46).

\section{References}

1. Hijal T, Fournier-Bidoz N, Castro-Pena P, Kirova YM, Zefkili S, Bollet MA, Dendale R, Campana F and Fourquet A: Simultaneous integrated boost in breast conserving treatment of breast cancer: A dosimetric comparison of helical tomotherapy and three-dimensional conformal radiotherapy. Radiother Oncol 94: 300-306, 2010.

2. Clarke M, Collins R, Darby S, Davies C, Elphinstone P, Evans V, Godwin J, Gray R, Hicks C, James S, et al; Early Breast Cancer Trialists' Collaborative Group: Effects of radiotherapy and of differences in the extent of surgery for early breast cancer on local recurrence and 15-year survival: An overview of the randomised trials. Lancet 366: 2087-2106, 2005.

3. Fisher B, Anderson S, Bryant J, Margolese RG, Deutsch M, Fisher ER, Jeong JH and Wolmark N: Twenty-year follow-up of a randomized trial comparing total mastectomy, lumpectomy, and lumpectomy plus irradiation for the treatment of invasive breast cancer. N Engl J Med 347: 1233-1241, 2002.

4. Kong FM, Klein EE, Bradley JD, Mansur DB, Taylor ME, Perez CA, Myerson RJ and Harms WB: The impact of central lung distance, maximal heart distance, and radiation technique on the volumetric dose of the lung and heart for intact breast radiation. Int J Radiat Oncol Biol Phys 54: 963-971, 2002.

5. Kirova YM, Fournier-Bidoz N, Servois V, Laki F, Pollet GA, Salmon R, Thomas A, Dendale R, Bollet MA, Campana F, et al: How to boost the breast tumor bed? A multidisciplinary approach in eight steps. Int J Radiat Oncol Biol Phys 72: 494-500, 2008.

6. Gray JR, McCormick B, Cox L and Yahalom J: Primary breast irradiation in large-breasted or heavy women: Analysis of cosmetic outcome. Int J Radiat Oncol Biol Phys 21: 347-354, 1991.

7. Neal AJ, Mayles WP and Yarnold JR: Invited review: Tangential breast irradiation-rationale and methods for improving dosimetry. Br J Radiol 67: 1149-1154, 1994.

8. Moody AM, Mayles WP, Bliss JM, A'Hern RP, Owen JR, Regan J, Broad B, Yarnold JR: The influence of breast size on late radiation effects and association with radiotherapy dose inhomogeneity. Radiother Oncol 33: 106-112, 1994

9. Neal AJ, Torr M, Helyer S and Yarnold JR: Correlation of breast dose heterogeneity with breast size using 3D CT planning and dose-volume histograms. Radiother Oncol 34: 210-218, 1995.

10. Kestin LL, Sharpe MB, Frazier RC, Vicini FA, Yan D, Matter RC, Martinez AA and Wong JW: Intensity modulation to improve dose uniformity with tangential breast radiotherapy: Initial clinical experience. Int J Radiat Oncol Biol Phys 48:1559-1568, 2000.

11. Vicini FA, Sharpe M, Kestin L, Martinez A, Mitchell CK, Wallace MF, Matter R and Wong J: Optimizing breast cancer treatment efficacy with intensity-modulated radiotherapy. Int J Radiat Oncol Biol Phys 54: 1336-1344, 2002.

12. Lo YC, Yasuda G, Fitzgerald TJ and Urie MM: Intensity modulation for breast treatment using static multi-leaf collimators. Int J Radiat Oncol Biol Phys 46: 187-194, 2000

13. Palma DA, Verbakel WF, Otto K and Senan S: New developments in arc radiation therapy: A review. Cancer Treat Rev 36: 393-399, 2010.

14. Lee TF, Ting HM, Chao PJ and Fang FM: Dual arc volumetric-modulated arc radiotherapy (VMAT) of nasopharyngeal carcinomas: A simultaneous integrated boost treatment plan comparison with intensity-modulated radiotherapies and single arc VMAT. Clin Oncol (R Coll Radiol) 24: 196-207, 2012.
15. Bertelsen A, Hansen CR, Johansen J and Brink C: Single arc volumetric modulated arc therapy of head and neck cancer. Radiother Oncol 95: 142-148, 2010.

16. Chan OS, Lee MC, Hung AW, Chang AT, Yeung RM and Lee AW: The superiority of hybrid-volumetric arc therapy (VMAT) technique over double arcs VMAT and 3D-conformal technique in the treatment of locally advanced non-small cell lung cancer - a planning study. Radiother Oncol 101: 298-302, 2011.

17. Quan EM, Li X, Li Y, Wang X, Kudchadker RJ, Johnson JL, Kuban DA, Lee AK and Zhang X: A comprehensive comparison of IMRT and VMAT plan quality for prostate cancer treatment. Int J Radiat Oncol Biol Phys 83: 1169-1178, 2012.

18. Kopp RW, Duff M, Catalfamo F, Shah D, Rajecki M and Ahmad K: VMAT vs. 7-field-IMRT: Assessing the dosimetric parameters of prostate cancer treatment with a 292-patient sample. Med Dosim 36: 365-372, 2011.

19. Guckenberger M, Richter A, Krieger T, Wilbert J, Baier K and Flentje M: Is a single arc sufficient in volumetric-modulated arc therapy (VMAT) for complex-shaped target volumes? Radiother Oncol 93: 259-265, 2009.

20. Bzdusek K, Friberger H, Eriksson K, Hårdemark B, Robinson D and Kaus M: Development and evaluation of an efficient approach to volumetric arc therapy planning. Med Phys 36: 2328-2339, 2009.

21. Solin LJ, Gray R, Goldstein LJ, Recht A, Baehner FL, Shak S, Badve S, Perez EA, Shulman LN, Martino S, et al: Prognostic value of biologic subtype and the 21-gene recurrence score relative to local recurrence after breast conservation treatment with radiation for early stage breast carcinoma: Results from the Eastern Cooperative Oncology Group E2197 study. Breast Cancer Res Treat 134: 683-692, 2012.

22. Zagouri F, Liakou P, Bartsch R, Peccatori FA, Tsigginou A, Dimitrakakis C, Zografos GC, Dimopoulos MA and Azim HA Jr: Discrepancies between ESMO and NCCN breast cancer guidelines: An appraisal. Breast 24: 513-523, 2015.

23. Wong EK, Truong PT, Kader HA, Nichol AM, Salter L, Petersen R, Wai ES, Weir L and Olivotto IA: Consistency in seroma contouring for partial breast radiotherapy: Impact of guidelines. Int J Radiat Oncol Biol Phys 66: 372-376, 2006.

24. Feuvret L, Noël G, Mazeron JJ and Bey P: Conformity index: A review. Int J Radiat Oncol Biol Phys 64: 333-342, 2006.

25. Ayata HB, Güden M, Ceylan C, Kücük N and Engin K: Comparison of dose distributions and organs at risk (OAR) doses in conventional tangential technique (CTT) and IMRT plans with different numbers of beam in left-sided breast cancer. Rep Pract Oncol Radiother 16: 95-102, 2011.

26. Jones R, Yang W, Read P and Sheng K: Radiation therapy of post-mastectomy patients with positive nodes using fixed beam tomotherapy. Radiother Oncol 100: 247-252, 2011.

27. Hurkmans CW, Meijer GJ, van Vliet-Vroegindeweij C, van der Sangen MJ and Cassee J: High dose simultaneously integrated breast boost using intensity modulated radiotherapy and inverse optimization. Int J Radiat Oncol Biol Phys 66: 923-930, 2006

28. van der Laan HP, Dolsma WV, Maduro JH, Korevaar EW, Hollander $M$ and Langendijk JA: Three-dimensional conformal simultaneously integrated boost technique for breast conserving radiotherapy. Int J Radiat Oncol Biol Phys 68: 1018-1023, 2007.

29. Singla R, King S, Albuquerque K, Creech S and Dogan N: Simultaneous integrated boost intensity modulated radiation therapy (SIB-IMRT) in the treatment of early stage left sided breast carcinoma. Med Dosim 31: 190-196, 2006. 